\title{
Design and Development of a Diabetes Self-Management Platform: A Case for Responsible Information System Development
}

\author{
Shane Joachima, ${ }^{\text {* }}$, Prem Prakash Jayaraman ${ }^{\mathrm{a}}$, Abdur Rahim Mohammad Forkan ${ }^{\mathrm{a}}$, Ahsan Morshed ${ }^{\mathrm{c}}$, Nilmini \\ Wickramasinghe ${ }^{\mathrm{a}, \mathrm{b}}$

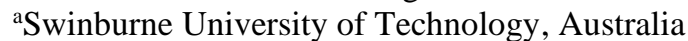 \\ ${ }^{b}$ Epworth HealthCare, Victoria, Australia \\ ${ }^{c}$ Central Queensland University, Australia \\ sjoachim@swin.edu.au
}

\begin{abstract}
Diabetes Mellitus a prevalent chronic disease that affects people from all genders and ages, continues to grow exponentially with predictions of nearly 578 million people affected by 2030. Self-management, known to be an essential aspect of any care program, can help patients with diabetics to control blood glucose and thereby, reduce the impact and likely complications. However, self-management to date has included the development of digital health solutions which have poor sustained uptake. This is primarily since such digital solutions have a poor fit with patient and clinician needs. In this paper, we propose a digital platform for supporting patients with diabetes. The proposed platform is a work-in-progress research and has been co-designed and co-developed (jointly with patients and clinicians) based on design science principles and includes key aspects of task-technology fit information system theory for further evaluation.
\end{abstract}

Keywords: Diabetes, self-management, online education, Task Technology Fit, Design Principle.

\section{Introduction}

Diabetes (Diabetes Mellitus), a prevalent chronic disease that continues to affect people across all genders and ages. 425 Million people were affected by diabetes (type I and II combined) in 2017 while this rapidly increased to 463 Million in 2019 [1]. This number is expected to grow to at least 578 million by 2030 [2] due to a combination of issues including drastic change of lifestyle, diet and lack of regular exercise [3]. In Australia, as of 2017-18 one in twenty Australians (4.9\% or over 1.2 million individuals) have diabetes and this figure continues to grow [4]. If this growth continues, up to 3 million Australians over the age of 25 will have diabetes by the year 2025 and 3.5 million by 2033 (with Type-II diabetes accounting for $85 \%$ of all diabetes) [5]. According to recent COVID-19 surveillance data reported by centre for diseases control (CDC), USA [6] the second most common underlying chronic health condition among COVID-19 patients was diabetes (30\%).

The complications of diabetes include damage to: (i) the large blood vessels leading to heart attack, stroke or circulation problems in the lower limbs; (ii) the small blood vessels causing problems in the eyes, kidneys, feet and nerves and (iii) issues with the skin, teeth and gums [7] thus making it an unpleasant chronic condition that requires further invasive, ongoing and expensive healthcare attention if left unchecked.

A key aspect in treating diabetes and its consequences, especially in the absence of any effective cure, is maintaining appropriate blood glucose levels by focusing on appropriate diet, physical activity, necessary medication management and regular screening [8]. A fundamental factor in adhering to a healthy lifestyle is to empower patients with diabetes to actively engage in self-management regimens [9]. A good self-management regimen can help avoid unnecessary and nasty complications that can develop due to uncontrolled diabetes while in some cases can even effectively help permanently reverse type II diabetes [8].

Self-management regimens for diabetes generally involve daily monitoring of blood glucose levels and blood pressure and keeping these within the patient's target ranges; eating a healthy diet focusing on foods with a low glycaemic index (GI); engaging in regular physical activity; reducing weight if it is above the recommended range and quitting smoking [7]. For example, increased physical activity alone is known to contribute to $30-50 \%$ reduction in the development of Type II diabetes [10]. Though self-management regimens can produce positive outcomes in managing

*corresponding author 
diabetes, based on recent research $[9,11]$, it has been demonstrated that the majority of people with diabetes find self-management regimens difficult to follow on an on-going basis.

Consequences of poor self-management regimens not only can cause potentially devastating outcomes for an individual but also puts enormous pressure on the healthcare system [1, 12]; e.g., in Australia, 40\% (\$55 billion) of healthcare costs are for chronic conditions while $\$ 2$ billion of that is paid by private health insurers. The additional cost incurred by individuals and government due to diabetes is significant and increases significantly in those patients with complications. These costs could be significantly reduced by providing patients with a better solution to adhere to diabetes self-management routines that can prevent the development of diabetes or its complications.

As stated earlier, self-management of diabetes is challenging for individuals. A recent survey [11] conducted with over 100 patients with diabetes has alluded to the need for a technological solution as an enabler for better diabetes self-management. Such a digital solution could be used also as an intervention tool to alleviate the challenges in diabetes selfmanagement. Hence, there is an immediate need to develop technological solutions that can support and provide educational reinforcement to people with diabetes in self-management.

While digital health solutions (including a plethora of mobile applications) for diabetic selfmanagement (predominately Type-II) exists it is evident from a recent review of such digital solution $[13,14]$ ambiguity among the solutions wide variability in key features pose significant difficulties for patients when using these solutions. A vast majority of these solution have been developed with minimal or no consultation with patients and clinicians hampering uptake of such solution among larger population of patients with diabetes. Further, the authors [13] emphasize the need for a co-designed solution involving patients, clinicians and policymakers.

Considering the above discussion and to answer the research question: "How can we responsibly develop an effective technological solution for selfmanagement of diabetes?", we present our work-inprogress research - a Diabetes Self-Management Platform. Our proposed platform aims to empower patients with a digital solution for better selfmanagement of diabetes. We opt the design science research methodology (DSRM) $[15,16]$ to co-design and co-development the platform. The co-design process includes eliciting key requirements from users (patients and clinicians) and validating the platform design with the users as per DSRM guidelines. Furthermore, we use a well-known information system theory - Task Technology Fit model [17] as our guiding theoretical lens in evaluating the platforms fit for the given purpose of empowering patients with better self-management of diabetes.

\section{Background}

\subsection{Type II Diabetes}

Type II Diabetes, is the most common form of diabetes, and it accounts for around $90 \%$ of all diabetes worldwide [18]. In Type II diabetes, when the body cells begin to not respond to insulin, it makes the individual insulin resistant, and this state is called Hyperglycaemia [10]. There are many similarities regarding symptoms between Type I and Type II diabetes such as excessive thirst, blurry vision, frequent urination, unexplained weight loss etc [10]. In other scenarios, it is not uncommon for individuals to go completely without symptoms. Such variance in the likely hood of diagnosing Type II diabetes at first sight has resulted in one-third to one-half of the population with Type II diabetes undiagnosed for a long period of time [10]. Chances of developing Type II diabetes have been strongly correlated with the factors such as being overweight (clinical obesity), increasing age, ethnicity and many lifestyle factors such as physical inactivity, smoking and alcohol consumption [10].

The recommended treatment for patients developing or with Type II diabetes is an effective self-management regime that can provide the necessary interventions on current lifestyle habits [7]. Encouraging and empowering individuals to be more physically active, practice healthy diet and provide educational reinforcement on an ongoing basis is critical for a successful self-management routine [19].

\subsection{Theoretical Framework: Task Technology Fit}

The Task Technology Fit (TTF) model [17, 20], is a well-known theory that has been used to guide the fit for purpose evaluation of information systems. According to Goodhue and Thompson [17] - "IT is more likely to have a positive impact on individual performance and be used if the capabilities of the IT match the tasks that the user must perform"(p.216). Technology is defined as a technological solution that comprises of hardware, software, and data flows to facilitate users to accomplish their tasks [17]. The TTF model for the whole context refers to the degree which a technology assists an individual in performing his or her portfolio of tasks [17,19]. 
Some research on TTF [21-25] has been carried out on extending the TTF model to the context of different information systems such as group support system, database management systems, software maintenance tools and wireless technology, e-health records. For example, [23] considered a fit-viability model to study m-commerce applications. In their framework, viability measures the readiness of the organisation for the technology adoption and implementation, and fit measures the capabilities of the systems to optimally perform the required tasks [22]. Particularly, in [24], Park et.al., illustrated an idea on content characteristics as a new determinant of fit that can contribute to the explanatory power of the TTF model. Content is defined here as all forms of knowledge, information, and data [23].

Figure 1 identifies factors which help build context around the identified tasks. The identified factors from a high level are external, organisation and individual factors (healthcare professionals and patient context).

External factor (Australian Healthcare Context) recognizes the factors that impact all the other 'factors' to follow. The locality of this research is within the Australian Healthcare System. This would require all parties to comply by the regulations set by the Australian Healthcare System, which in return effect the fit of all identified tasks and technologies.

Organisation factors identify influences caused by the clinic or hospital. These influences for example are things such as operation policies and staff training. Where differences in set policies and providing staff training will impact the fit of the clinical support task and related technology.

Individual factors are characteristics of the two user groups, patient, and healthcare professionals. The identified characteristics consider the individuals background and capabilities which ultimately correlate with their experience regarding medication,

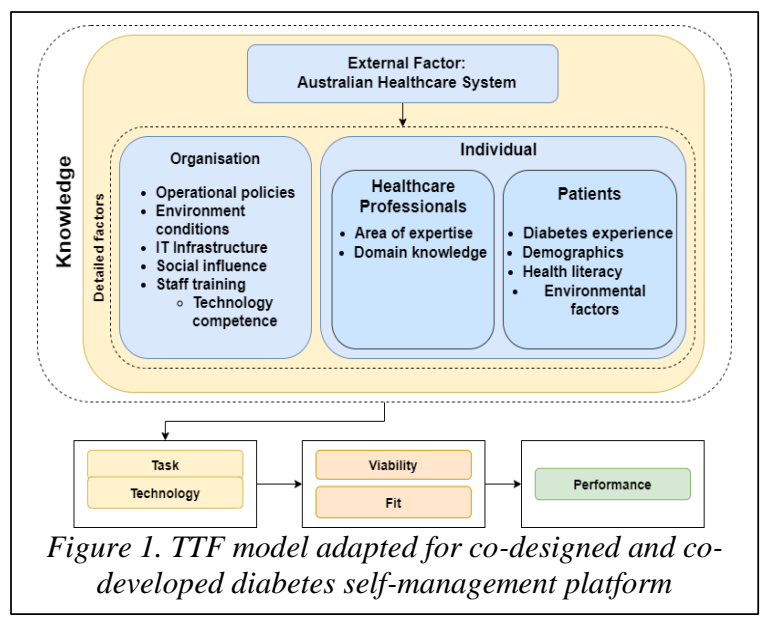

blood glucose monitoring, fitness performance monitoring and nutrition tasks.

Task and Technology will be measured based on $[20,26]$ and also Fit will be measured by matching the requirements of the organisation with the functionalities offered by the system e.g. data format, operating procedures, and output format as well as other successful translative performance factors such as timeliness, reliability and accuracy [27].

\subsection{Methodology - DSRM}

Design Science Research Methodology (DSRM) revolves around the aim of building multiple sociotechnical artefacts, which range from software, processes, computer algorithms and systems with the goal to improve and/or solve the problem at hand [2830]. Further, Hevner and Wickramasinghe [31] employed DSRM to healthcare contexts, noting the importance of the adapting and using of DSRM in healthcare contexts when patient-centric solutions are a priority. Given this, DSRM was incorporated into our methodology. Particularly, we followed the seven guidelines proposed by [15] for understanding, executing, and evaluating design science research. Various studies [32-34] have used these guidelines for building algorithms and systems.

The four-cycle model [16] was utilized to compose the actions required for the co-design phases of the project. The application of the DSRM model is as follows:

The Change and Impact cycle: ensures that the designed solution would be fit for purpose in the Australian Healthcare context. Items that were considered was the designed solution, the mobile devices(s) used and the patients and/or clinicians which may use the solution.

The Relevance Cycle: involves the identification of key requirements of the users (patients and clinicians) by grasping the problems faced in their environment through a range of discussions such as interviews, focus groups and other techniques.

The Co-Design Cycle I, II \& III: refers to the design and development of any artefacts that are produced. Which include items such as the paper prototype and diabetes management platform itself. This cycle ensures that the artefacts go through a range of evaluation strategies which ensure the nature of the solution caters the problem domain as intended.

The Rigor Cycle: enables us to verify and populate the knowledge base with our findings and contributions to the space. From the artifacts designed and developed in prior cycles, we extract the contributions which range from scientific theories, artefact evaluations - capturing what works and what does not, but also experience and expertise [16]. 


\section{Related Applications}

In the space of digital health applications, there are currently more than 300,000 mobile apps available for a user to download: with diabetes management applications being the most [12]. Out of the applications, for this study, we have chosen ten applications from both Apple's App Store and Google's Play Store for analysis. The apps were selected based on some key factors. They are I) Total user downloads, II) Standard feature set, III) Standout unique features, IV) Must have a free tier. With this criterion in mind, keywords such as "diabetes", "management", "self-management", "adherence" were queried in the respective stores. With a select list of apps filtering through the set criteria, we further manually handpicked ten apps based on positive comments and overall higher rating. Further needs to be highlighted that since Apple's App Store does not publicly present the total number of application downloads, the total number of ratings were taken into consideration.

To manage Type II diabetes, the requirement is to follow a healthier lifestyle by controlling a range of factors such diet, physical activities, and medications. These factors can be extracted to a high-level feature set category. The identified common feature sets with all the applications can be divided into the following categories: Medication, Blood Glucose, Fitness, Nutrition and Clinical. Figure 2 provides an overview of all the selected apps, and the aggregated results of how they performed in each of the feature set categories. For each of the feature set category, many specific features were identified as key influencer of diabetes. These features were averaged to populate Figure 2. More detailed analysis on those features can be found in the Preliminary Results section 6.2.

Type II diabetes is typically directly correlated with poor lifestyle and nutrition management [18], yet none of these applications cater for these areas completely. Through a comparative gap analysis, we found that there are clear gaps in the areas of Clinical, Nutrition and Fitness to be addressed. As there are applications such as 'Glucose buddy diabetes tracker' which cater for Fitness features and 'Diabetes: $M$ ' that comprehensively cover the clinical features, there is no single personalised diabetes self-management application that covers all the vital features outlined as a part of this review. In addition, we found that none of these solutions catered for cultural or ethnic nuances either. The applications compared in this review, was chosen due to popularity and demand; however, none of them are linked to any formal clinical study and do not contain sufficient clinical support features. This further highlights the lack of responsible development initiatives put in place while designing and developing diabetes self-management applications, which could be one of the biggest factors around the lack of "completeness" regarding application features. Hence, this clearly highlights the importance to include the user's perspective. Thus, we take a Design Science Research Methodology (DSRM) approach to design and develop a personalised diabetes platform validated through rigorous evaluation strategies, to address a key void in diabetes self-management care support. Figure 2 depicts the feature set that are best covered by the top applications in the current market. The legend for this

\begin{tabular}{|c|c|c|c|c|}
\hline \multirow[b]{2}{*}{ Name } & \multicolumn{4}{|c|}{ Feature set } \\
\hline & $\begin{array}{l}3 \\
0 \\
0 \\
\vdots \\
0 \\
\end{array}$ & 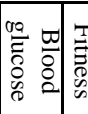 & & 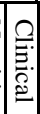 \\
\hline MySugr & & & & \\
\hline Blood Sugar Log & & & & \\
\hline Glucose Tracker \& Diabetic Diary & & & & \\
\hline Diabetes:M & & & & \\
\hline Glucose buddy diabetes tracker & & & & \\
\hline One drop diabetes management & & & & \\
\hline Blood sugar monitor by Dario & & & & \\
\hline Blood Glucose Tracker & & & & \\
\hline forDiabetes:diabetes self-management app & & & & \\
\hline Glucose - blood sugar tracker (iOS only) & & & & \\
\hline
\end{tabular}

Figure 2 is as follows: Green: All required features exist; Orange: Required features partially exist; Red: Required features missing without replacement.

\section{Diabetic Self-Management Platform: Adopting DSRM for Co-design}

In this section we present the DSRM guidelines and DSRM cycles, adapted to the context of responsibly designing and developing a diabetic selfmanagement platform.

\subsection{DSRM Guidelines}

Guideline 1: Design as an Artefact: A diabetes management platform which caters for both patients and clinicians (e.g. nurses) and allows for selfmanagement of a patient's diabetes journey. This could strengthen the quality of care and timeliness of feedback a patient receives, yet not dramatically impact the cost of care delivery.

Guideline 2: Problem Relevance: Provision of continuous and superior monitoring and management of diabetes. Regardless of restrictions such as location and/or time, the patient and clinicians can access vital information promptly, allowing for improved decision making in relation to diabetes management; with a capable solution which is designed and developed to enable self-management of diabetes. 


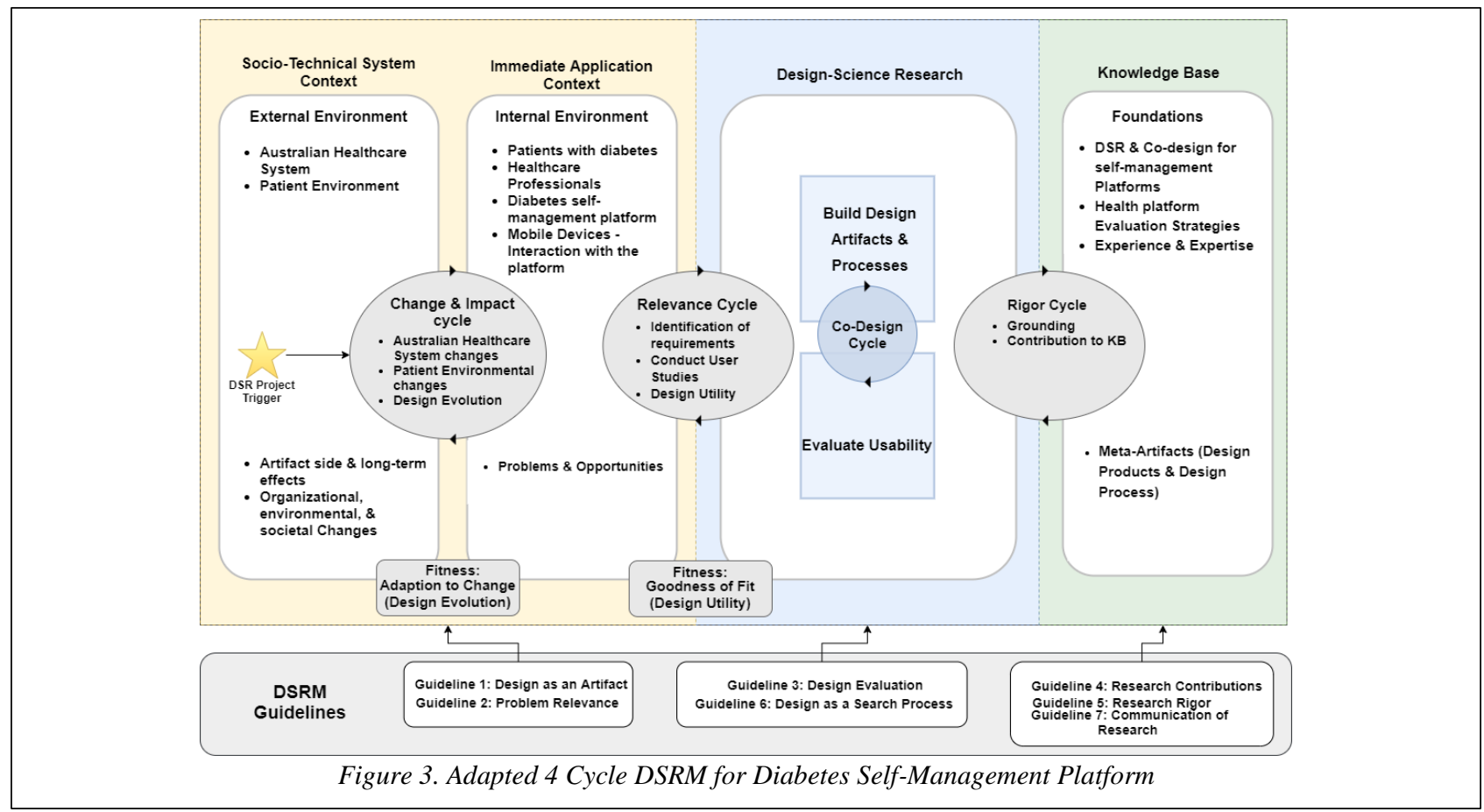

Guideline 3: Design Evaluation: Emphasis was placed with the conscious integration of potential patient users and clinicians at various stages of the design and evaluation stages of the solution. Further, senior representatives from supporting hospitals were consulted to ensure the proposed solution aligned with many of the government regulations and requirements for technology solutions interacting with patients in the context of medical research. Design Evaluation was an iterative process which reached completion when all parties (legal, clinical, patients etc.) were content with the artefact and is fit to serve purpose from their regard.

Guideline 4: Research Contributions: In this study, users' perspectives of the mediating role of the solution are explored.

Guideline 5: Research Rigor: Information systems conceptual models and theoretical groundings were employed. This allowed for existing chronic disease management protocols, healthcare quality and safety information were consulted to inform the development cycles to allow for a clinical context evaluation to take place.

Guideline 6: Design as a Search Process: For the ability to conduct a clinical trial and obtaining sensitive patient data, the design was strictly monitored to ensure the ethics requirements were met.

Guideline 7: Communication of Research: (I) Internal communication: Present the technology and clinically oriented users through focus groups, simulations exercises, brainstorming meetings, as well as technical and managerial meetings. (II) External communication: Progress and findings are to be reported in relevant peer review outlets including international conferences and professional peerreviewed journals in relevant disciplines.

\subsection{DSRM Cycles}

In this section we focus on the adoption and the processes of the 4 cycle DSRM [16]. Figure 3 represents the adapted version of the 4 cycle DSRM, where the context is designing, developing, and evaluating the diabetes self-management platform.

4.2.1. Change \& Impact (CI) Cycle allows us, with the External Environmental Factors (Australian Healthcare \& Patient Environment) in mind, to identify factors which can influence patients and clinicians in a wider context. This was achieved by collaborating with multiple experienced Australian healthcare professionals with foundations in diabetes.

Further, the CI Cycle, enables the validation of the designed artefacts to ensure that the research grounding effecting the above-mentioned factors are still monitored. This validation also ensures that if any core changes are made to the process or state of the artefact, the External Environment Factors are reevaluated to ensure the solution is fit for purpose. This is made possible due to the incorporation of the Design Evolution Fitness Model.

4.2.2. Relevance Cycle Helps with the identification of the key requirements that are deemed critical for a diabetic self-management solution. We started by identifying factors of the Internal 
Environment which directly influence a diabetes platform. The identified factors are: I) Patients with diabetes; II) Healthcare professionals; III) Diabetes self-management platform (proposed artefact); IV) Mobile devices used to interact with the platform.

A wide range of semi-structured interviews and workshops were conducted, with the participation of patients with diabetes and healthcare professionals. This ensures the identified requirements allow for improved management of a patient's diabetes. This cycle, through the incorporation of Design Utility Model, ensures the developed platform stays fit for purpose. The model enables a verification process which involves a join evaluation of the identified requirements and the user studies data, once conducted.

In one of the workshops, the group discussion adopted a problem-solving strategy 'Working backwards'. As the name suggests, the concept of this method is to start off with a large/desired end goal, and demystify the steps required to achieve that goal by working backwards [35]. This activity was run iteratively over a predefined set of key objectives and topics that were noted as most important by healthcare domain experts. The results are presented in Table 1.

A wide range of semi-structured interviews and workshops were conducted, with the participation of patients with diabetes and healthcare professionals from a hospital in Victoria, Australia. This ensures the identified requirements allow for improved management of a patient's diabetes.

This cycle, through the incorporation of Design Utility Model, ensures the developed platform stays fit for purpose. The model enables a verification process which involves a join evaluation of the identified requirements and the user studies data, once conducted.

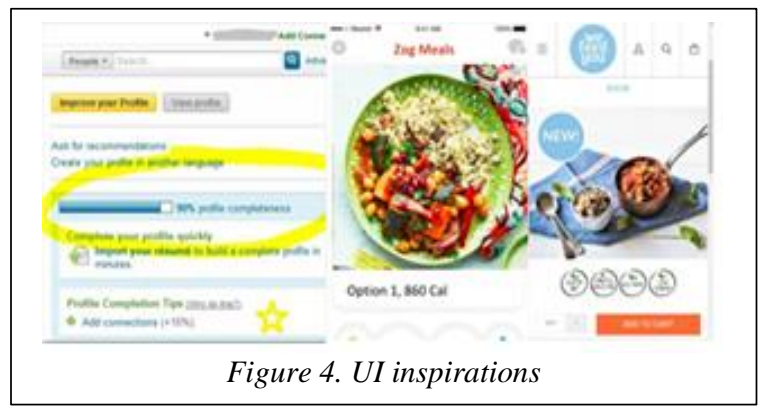

4.2.3. Co-Design Cycles is an interactive iterative process. Our project required numerous iterations, but they can be split up into three parts. This refers to the initial Design, followed by the Development and then the rigorous Evaluation of the artefact.

4.2.3.1. Co-Design Cycle $I$ is the first iteration of the design cycle, we development a set of mock-up pages. The mock-ups were inspired from various sources of existing application designs but were carefully adapted to meet the identified requirements of the diabetes self-management platform. Mock-up designs are presented in Figure $4 \& 5$. After the presentation of the mock-ups to the patients and clinicians, a discussion was prompted to better understand their thoughts on it (evaluation).

4.2.3.2. Co-Design Cycle II Interacts with the results that were collected from the evaluation following Co-Design Cycle I and allow for a revision of the original set of mock-ups to be made. The results reflected many red \& orange dominant sections of Figure 2. The evaluations made apparent the lack of personalization for both patients and clinicians, and it was added. For patients, this focused on features that enabled them to set meal preferences regarding their diet and exercise. Where in contrast, the clinicians

\begin{tabular}{|c|c|c|}
\hline \multicolumn{3}{|c|}{$\begin{array}{c}\text { Table 1. Clinician \& Patient Workshop Requirement } \\
\text { Identification }\end{array}$} \\
\hline Category & Task & Solution \\
\hline \multirow[t]{6}{*}{ Lifestyle } & $\begin{array}{l}\text { Searching for } \\
\text { meals }\end{array}$ & $\begin{array}{l}\text { Ability to find meals } \\
\text { based of name search. }\end{array}$ \\
\hline & Meal plans & $\begin{array}{l}\text { Picking a meal from a } \\
\text { defined meal plan. }\end{array}$ \\
\hline & $\begin{array}{l}\text { View meal } \\
\text { information }\end{array}$ & $\begin{array}{l}\text { View ingredients and } \\
\text { nutrition information of a } \\
\text { selected meal. }\end{array}$ \\
\hline & Log meals & $\begin{array}{l}\text { Add meals consumed to } \\
\text { log. }\end{array}$ \\
\hline & $\begin{array}{l}\text { Meal } \\
\text { preferences }\end{array}$ & $\begin{array}{l}\text { Ability to set culture } \\
\text { specific cuisines and } \\
\text { other preferences as } \\
\text { priority during search. }\end{array}$ \\
\hline & $\begin{array}{l}\text { Log fitness } \\
\text { activities }\end{array}$ & $\begin{array}{l}\text { Add any physical activity } \\
\text { with duration undertaken. }\end{array}$ \\
\hline $\begin{array}{l}\text { Medication } \\
\text { management }\end{array}$ & $\begin{array}{l}\text { Log } \\
\text { medication }\end{array}$ & $\begin{array}{l}\text { Add any medication } \\
\text { taken for a given day. }\end{array}$ \\
\hline \multirow[t]{3}{*}{ Resources } & $\begin{array}{l}\text { Type II } \\
\text { diabetes } \\
\text { information }\end{array}$ & $\begin{array}{l}\text { Provide FAQ information } \\
\text { from Diabetes Australia. }\end{array}$ \\
\hline & $\begin{array}{l}\text { Support } \\
\text { group }\end{array}$ & - \\
\hline & $\begin{array}{l}\text { Hospital } \\
\text { contact }\end{array}$ & $\begin{array}{l}\text { Provide contact details of } \\
\text { their hospital/GP from in } \\
\text { app. }\end{array}$ \\
\hline \multirow[t]{3}{*}{ Miscellaneous } & $\begin{array}{l}\text { General } \\
\text { Statistics }\end{array}$ & $\begin{array}{l}\text { To view how the patient } \\
\text { is tracking with their } \\
\text { diabetes journey. } \\
\text { - } \text { Avg, highest, } \\
\text { lowest } \\
\text { mmollL } \\
\text { - } \quad \text { Overall } \\
\text { progress }\end{array}$ \\
\hline & $\begin{array}{l}\text { Log blood } \\
\text { sugar levels }\end{array}$ & $\begin{array}{l}\text { Ability to } \log \mathrm{mmol} \backslash \mathrm{L} \\
\text { levels at a given time. }\end{array}$ \\
\hline & $\begin{array}{l}\text { View blood } \\
\text { sugar in an } \\
\text { interactive } \\
\text { chart }\end{array}$ & $\begin{array}{l}\text { A line chart which } \\
\text { contains all the blood } \\
\text { sugar entries for a given } \\
\text { timeframe. }\end{array}$ \\
\hline
\end{tabular}


identified the need of a simple visualization, which graphed the patient's glucose levels over a timeseries. A paper prototype was built in this cycle. This prototype acted as the successor to the revised mockups and addressed all the quires brought up during the evaluation of the previous cycle. The paper protype also introduced the User Interface (UI) for the platform in its entirety, in more detail. This also prompted a discussion from the patients and clinicians, which focused on evaluating the design and functional elements.

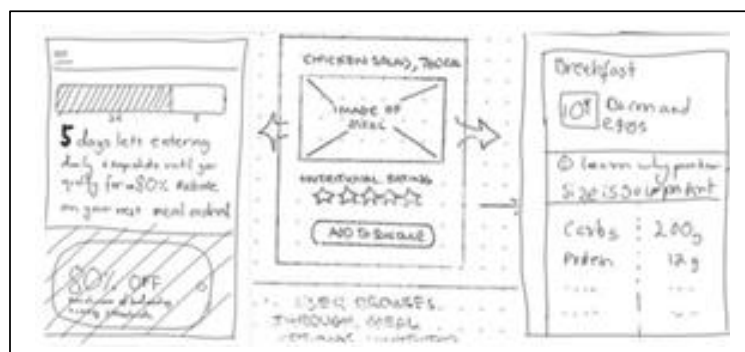

Figure 5. Mock-up based on inspirations

4.2.3.3. Co-Design Cycle III Following the paper prototype evaluations with the patients and clinicians, the design and the functional flow of the platform was finalised. From here, works for the development of the functional platform (artefact) was started. Throughout the development process, a bi-weekly meeting was scheduled with the healthcare professionals and stakeholders to provide input of the current state of the platform at that given point in time. This further ensured that the development process was continuously validated, allowing for the artefact to address the problem domain as intended. Figure 6 contains an aspect of the platform, that is designed and developed for the patients.

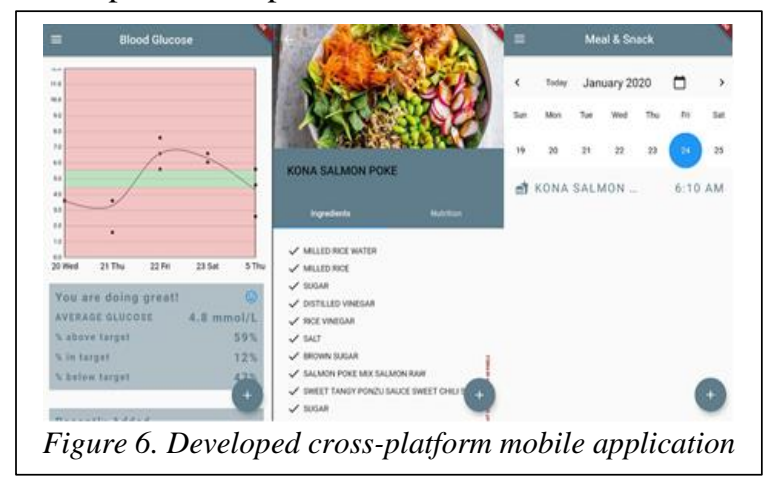

4.2.3.4. Rigor Cycle Enabled us to view and verify our contributions to this domain, ensuring that we are building and contributing something novel. Our contribution through this project was directed at the usage of DSRM \& Co-design for responsible design and development of a diabetes management platform. With that in mind, we were able to validate to further validate the UI elements, improved evaluation strategies when it comes to evaluating diabetes platforms and general contributions to Experience \& Expertise.

\section{Preliminary Outcome}

Using DSRM for Co-design and Co-development proved to be crucial in building the requirements for this self-management platform. This accounts for both the functional platform and the evaluation purposes.

\subsection{Implementation}

Following the DSRM, we identified the implementation for the diabetes self-management platform will have to come in two ways. To see that there are two very different user groups, patients, and healthcare professionals, it was important to limit functionality between a clinical user and a general user. Hence, through the Relevance cycle, we identified each user groups essentially require their own implementation. A cross platform mobile application for the patients and a web application for the healthcare profession (nurse/clinician portal).

With that in mind, we went ahead and implemented a full stack system. For the frontend, the platform consists of a cross platform mobile application, targeting Android and iOS, built using Flutter SDK; and for the nurse/clinician portal, we have implemented a single page application (SPA) using Vue.js. The two frontend systems are connected to a NodeJS application programming interface (API), where the emphasis of the design and implementation was on speed, robustness, and security. The backend connects to a $M y S Q L$ database which is generated by the API. Figure 7 illustrates this structure.

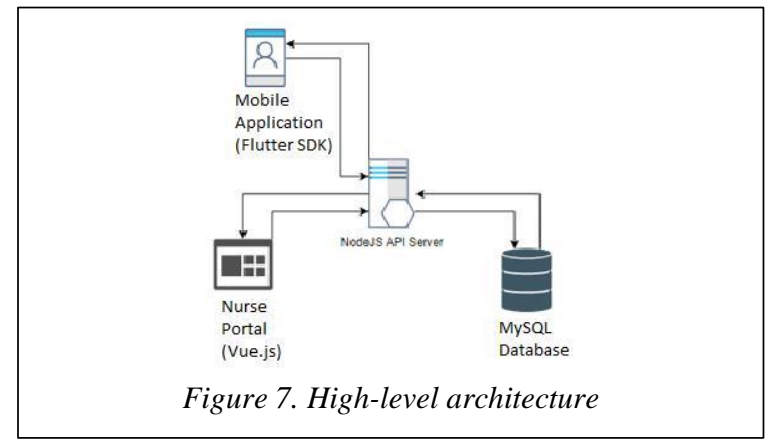

Further, the mobile application and nurse portal is locked and secured. Using a Json Web Token (JWT) system with an authentication middleware, checking requests, and Argon2 hashing algorithm to handle and store passwords in a secure manner. Since we are working with sensitive data, the API has been setup to produce tokens with ' $8 h$ ' expiration for the mobile application and ' $30 d$ ' expiration for the nurse/clinician portal. This will require users to login into the application with a predefined username and password, 
which they can modify after being issued an API generated one the first time.

\subsection{Preliminary Findings}

The utilisation of co-design and DSRM together provided various improvements to the identification of the requirements as well as the strategies used to evaluate this platform. The addition of the co-design principal, with our adapted DSRM model, we were able to integrate clinicians/healthcare professionals and patients throughout the DSRM Cycles. This allowed for two different perspectives on the same platform. The addition of clinicians and healthcare professionals results in that the evaluation of the platform differs immensely compared to previous traditional methods, which would have only had the directly affected actor (patients) evaluate the platform. The clinicians were able to provide professional and clinical input with the identification of key requirements and the evaluation of the platform, which eliminates any medical or clinical oversights. This also means that the platform is built with a clinical grounding, with in returns grants the platform to be clinically sound and medically safe.

Another benefit in integrating the two user groups, patients, and healthcare professionals, is that this further enables us to verify that the diabetes selfmanagement platform application is inline of the user's expectation.

Further, this integration process of both clinicians/healthcare professionals and patient contexts provides the ability to initiate a translation process. This involves taking the clinician requirements and patients inputs and consolidating them into a single unique list of requirements. Which will reflect both functional and UI requirements. This promises that the final platform will result in a single unified, patient-centric self-management solution, which is also highly personalised to the user group's needs. By taking the approach of integrating clinical and patient contexts with DSRM, we are ensured that the platform being designed and developed, is being done in a responsible manner.

Here we discuss further regarding the task and technology characteristics, discussed earlier in Theoretical Framework: Task Technology Fit section 2.2 .

We present the identified task and technology characteristics for our diabetes self-management platform and an evaluation of the fit of how our platform compares to the other solutions identified in Figure 2. This task list also consistent with DSRM Relevance cycle gathered through workshops involving patients with diabetes and clinicians. Each task contains identified technological characteristics.
Overall, the comparative gap analysis performed in section 3 and figure 2 suggests that most, if not all, existing approaches have missed some technologies that were noted as key requirements for corresponding task. Thus, making clear the importance of taking a responsible approach to designing and developing IS solutions to minimise these critical oversights.

\subsubsection{Task-1: Medication Management}

- Store medication history: Ability to track any intake of medication including dosages by storing information of every medication intake.

- Search medication: Ability to search and select a medication from an authentic medication database validated by clinicians.

- Create medication intake: Ability to create a medication intake based on customised user input (e.g. medication name, dosage).

Evaluation: $80 \%$ of the reviewed applications had technology to over store medication history and create medication intake. However only $\mathbf{1 0 \%}$ supported search medication.

\subsubsection{Task-2: Blood Glucose level monitoring}

- Store blood glucose levels: A method to selfmonitor blood glucose with provision to store each blood glucose measurement along with time.

- Visualise blood glucose: Ability to visualise previously stored blood glucose data using suitable graphs with filtering capability.

- Goal settings for blood glucose level: Ability to set a minimum and maximum value of blood glucose level to monitor the progress of diabetes management.

- View statistics of Blood glucose: Ability to view insightful information based on the historical blood glucose data.

Evaluation: Blood Glucose level Monitoring task is well covered. With $\mathbf{1 0 0 \%}$ - all the reviewed applications have technology to support store blood glucose level, visualise blood glucose and view statistics of blood glucose. Further $\mathbf{8 0 \%}$ contained technology to support to goal setting for blood glucose levels.

5.2.3. Task-3: Fitness performance monitoring

- Store fitness activity: A method to keep track and store fitness activities.

- $\quad$ Create personalised activity: Ability to create a customised firstness activity with relevant fields such as type, duration, and intensity.

- Estimate fitness performance: A functionality to compute fitness performance by estimating total calories burned for the planned activities.

Evaluation: Fitness performance monitoring was poorly covered. With only $\mathbf{3 0 \%}$ of the reviewed applications allowed for you to store fitness activity; $\mathbf{2 0 \%}$ has the technology to create personalised activity 
and $\mathbf{1 5 \%}$ with the technology to estimate fitness performance.

\subsubsection{Task-4: Nutrition planner}

- $\quad$ Store nutrition: A method to keep track and store of nutritional content intake.

- $\quad$ Search nutritional contents: Ability to search for meals, drinks, snacks etc, through an authentic resource using internet search.

- Create nutritional content: Ability to manually create and store consumed meal items.

- View nutritional information on meals: A feature which displays a comprehensive list of nutritional information (ingredients, kcal, protein etc.) based on a selected meal.

- Store planned meal: A feature that allows to create and store a list of pre-planned meals.

- Recommend for nutrition: A personalised nutrition recommender feature, which suggests meals based on a range of parameters (e.g. meal preference, calory amount).

Evaluation: Nutrition planner was represented poorly, with clear oversights to some tasks. $65 \%$ of the reviewed applications has technology to store nutrition; $\mathbf{6 0 \%}$ contains technology to search nutritional contents; $35 \%$ contains technology to search nutritional contents; $\mathbf{3 0 \%}$ contains technology to view nutritional information on meals; $5 \%$ offering technology to store planned meals; $0 \%$ - none of the reviewed applications had technology to recommend for nutrition.

\subsubsection{Task-5: Clinical support}

- Contact support: A method for the patients to reach out experts (e.g. diabetes coach) for further supports and advices.

- Remote Monitoring: Ability for clinicians to monitor and view the progress of patients using remote mechanism (nurse/clinician portal).

- View assigned individuals' details: A feature which enables the patient or healthcare professional to view the details of the individuals they are assigned to through the platform.

Evaluation: Clinical support was almost non-existent. With $\mathbf{1 0 \%}$ of the reviewed applications offered technology for remote monitoring and view assigned individual's details; $20 \%$ offered paid technology to enable contact support, if not considering paid features, then $0 \%$ offered this technology.

\section{Conclusion \& Future Works}

This work in progress research reported on the outcomes of a co-design and co-developed platform for self-management of diabetes. Our proposed platform was co-designed with patients and clinicians and we used the DSR methodology to elicit requirements from the users. We then employ TTF model to assess and evaluate the "fit for purpose" of the developed solution. This far, the research has completed validating a paper prototype with clinicians and patients. A first iteration of the implemented platform presented in the paper has been validated with clinicians and as part of our future work outlines below, we aim to validate the platform with a cohort of patients with diabetes. Next steps include a plan to address any gaps found in the nutrition support in the platform. Currently, it meets all the basic requirements of the users, we plan on making this smart and personalised to the user. The aim of this work is to help determine whether the platform can provide improved and sustained ongoing support, and better glucose control, for patients with type 2 diabetes, and nutrition is a large part of that. We will also be focusing on pilot-testing and evaluating the developed platform to establish proof of concept with three target groups of patients with type 2 diabetes, drawn from Caucasian, Indian and Muslim communities. This choice of cohort has been made to allow for diverse diet and exercise options to assess the personalisation feature, tailored to distinctively diverse ethnic dietary practices. We have identified a hospital in Victoria as a partner for recruiting patient cohorts.

\section{Acknowledgements}

We are very grateful for the support and input provided by Dr John Zelcer and Professor Peter Brooks. We also thank Norther Health and all the patients \& clinicians who participated in the study.

\section{References}

[1] N. H. Cho et al., "IDF Diabetes Atlas: Global estimates of diabetes prevalence for 2017 and projections for 2045," Diabetes Research and Clinical Practice, vol. 138, pp. 271-281, 2018, doi: 10.1016/j.diabres.2018.02.023.

[2] P. Saeedi et al., "Global and regional diabetes prevalence estimates for 2019 and projections for 2030 and 2045: Results from the International Diabetes Federation Diabetes Atlas, 9," Diabetes Research and Clinical Practice, vol. 157, 2019, doi: 10.1016/j.diabres.2019.107843.

[3] A. T. Kharroubi et al., "Diabetes mellitus: The epidemic of the century," (in eng), World J Diabetes, vol. 6, no. 6, pp. 850-867, 2015, doi: 10.4239/wjd.v6.i6.850.

[4] Australian Institute of Health and Welfare, "Diabetes," 15 July 2020. [Online]. Available: https://www.aihw.gov.au/reports/diabetes/diabetes/co ntents/how-many-australians-have-diabetes

[5] D. J. Magliano et al., "Projecting the burden of diabetes in Australia - what is the size of the matter?," Australian and New Zealand Journal of Public Health, vol. 33, no. 6, pp. 540-543, 2009, doi: 10.1111/j.17536405.2009.00450.x. 
[6] E. K. Stokes et al., "Coronavirus Disease 2019 Case Surveillance - United States, January 22-May 30, 2020," MMWR. Morbidity and Mortality Weekly Report, vol. 69, no. 24, pp. 759-765, 2020, doi: 10.15585/mmwr.mm6924e2.

[7] National Diabetes services scheme. "Diabetes-related complication." https://www.ndss.com.au/ (accessed 10 July, 2020).

[8] R. M. M. Khan et al., "From Pre-Diabetes to Diabetes: Diagnosis, Treatments and Translational Research," Medicina, vol. 55, no. 9, 2019, doi: 10.3390/medicina55090546.

[9] N. Wickramasinghe et al., "Achieving Value-Based Care in Chronic Disease Management: Intervention Study," JMIR Diabetes, vol. 4, no. 2, 2019, doi: 10.2196/10368.

[10] Y. Wu et al., "Risk Factors Contributing to Type 2 Diabetes and Recent Advances in the Treatment and Prevention," International Journal of Medical Sciences, vol. 11, no. 11, pp. 1185-1200, 2014, doi: 10.7150/ijms.10001.

[11] S. Rodda et al., "Enablers and barriers to effective diabetes self-management: A multi-national investigation," Plos One, vol. 14, no. 6, 2019, doi: 10.1371/journal.pone.0217771.

[12] C. M. Y. Lee et al., "The cost of diabetes in adults in Australia," Diabetes Research and Clinical Practice, vol. 99, no. 3, pp. 385-390, 2013, doi: 10.1016/j.diabres.2012.12.002.

[13] G. Jimenez et al., "Examining Diabetes Management Apps Recommended From a Google Search: Content Analysis," JMIR mHealth and uHealth, vol. 7, no. 1, 2019, doi: 10.2196/11848.

[14] E. Lum et al., "Decision Support and Alerts of Apps for Self-management of Blood Glucose for Type 2 Diabetes," Jama, vol. 321, no. 15, 2019, doi: 10.1001/jama.2019.1644.

[15] Hevner et al., "Design Science in Information Systems Research," MIS Quarterly, vol. 28, no. 1, 2004, doi: $10.2307 / 25148625$.

[16] A. Drechsler et al., "A four-cycle model of IS design science research: capturing the dynamic nature of IS artifact design," 2016.

[17] D. L. Goodhue et al., "Task-Technology Fit and Individual Performance," MIS Quarterly, vol. 19, no. 2, 1995, doi: 10.2307/249689.

[18] D. Federation, "IDF Diabetes Atlas 9th Edition," 2019.

[19] N. Wickramasinghe et al., "Achieving Value-Based Care in Chronic Disease Management: Intervention Study," JMIR Diabetes, vol. 4, no. 2, p. e10368, May 3 2019, doi: 10.2196/10368.

[20] D. L. Goodhue, "Development and Measurement Validity of a Task-Technology Fit Instrument for User Evaluations of Information System," Decision Sciences, vol. 29, no. 1, pp. 105-138, 1998, doi: 10.1111/j.1540-5915.1998.tb01346.x.

[21] E. Ammenwerth et al., "IT-adoption and the interaction of task, technology and individuals: a fit framework and a case study," BMC Medical
Informatics and Decision Making, vol. 6, no. 1, 2006, doi: 10.1186/1472-6947-6-3.

[22] S. D. Dishaw M, Bandy DB, "The impact of tasktechnology fit in technology acceptance and utilization models," presented at the AMCIS 2004 Proceedings, 2004.

[23] T. P. Liang et al., "Adoption of mobile technology in business: a fit-viability model," Industrial Management \& Data Systems, vol. 107, no. 8, pp. 1154-1169, 2007.

[24] C. Park, "Exploring a New Determinant of Task Technology Fit: Content Characteristics," Journal of International Technology and Information Management, vol. 27, no. 3, 2019.

[25] A. K. Tjan, "Finally, a way to put your Internet portfolio in order," (in eng), Harv Bus Rev, vol. 79, no. 2, pp. 76-85, 156, Feb 2001.

[26] A. Tariq et al., "An Assessment of M-Health in Developing Countries Using Task Technology Fit Model," in AMCIS, 2011.

[27] S. Chatterjee et al., "Examining the success factors for mobile work in healthcare: A deductive study," Decision Support Systems, vol. 46, no. 3, pp. 620-633, 2009, doi: 10.1016/j.dss.2008.11.003.

[28] M. D. Myers et al., "A set of ethical principles for design science research in information systems," Information \& Management, vol. 51, no. 6, pp. 801809, 2014, doi: 10.1016/j.im.2014.01.002.

[29] P. P. Jayaraman et al., "Healthcare 4.0: A review of frontiers in digital health," WIREs Data Mining and Knowledge Discovery, vol. 10, no. 2, 2019, doi: $10.1002 /$ widm. 1350 .

[30] A. Morshed et al., "Deep Osmosis: Holistic Distributed Deep Learning in Osmotic Computing," IEEE Cloud Computing, vol. 4, no. 6, pp. 22-32, 2017 , doi: $10.1109 / \mathrm{mcc} .2018 .1081070$.

[31] A. R. Hevner et al., "Design Science Research Opportunities in Health Care," in Theories to Inform Superior Health Informatics Research and Practice, (Healthcare Delivery in the Information Age, 2018, ch. Chapter 1, pp. 3-18.

[32] L. Nguyen et al., "An Examination of the Mediating Role for a Nursing Information System," Australasian Journal of Information Systems, vol. 21, 2017, doi: 10.3127/ajis.v21i0.1387.

[33] B. John et al., "Graph-based Cluster Analysis to Identify Similar Questions: A Design Science Approach," Journal of the Association for Information Systems, vol. 17, no. 9, pp. 590-613, 2016, doi: 10.17705/1jais.00437.

[34] D. Arnott et al., "A Critical Analysis of Decision Support Systems Research Revisited: The Rise of Design Science," Journal of Information Technology, vol. 29, no. 4, pp. 269-293, 2014, doi: 10.1057/jit.2014.16.

[35] M. Amit et al., "'Explicit teaching' as an effective method of acquiring problem solving strategies-the case of 'working backwards'," 2017. 